\title{
New Mathematical Model of Electromechanical Coupling in Rat Cardiomyocytes
}

\author{
Leonid B Katsnelson $^{1,2}$, Pavel Konovalov ${ }^{1}$, Olga Solovyova ${ }^{1,2}$ \\ ${ }^{1}$ Institute of Immunology and Physiology of the RAS, Ekaterinburg, Russia \\ ${ }^{2}$ Ural Federal University, Ekaterinburg, Russia
}

\begin{abstract}
The rat is one of the most widely used laboratory animal species. Therefore development of mathematical models aimed to analyze electromechanical coupling in the rat myocardium is a matter of top interest. We have developed a novel model of excitation-contraction coupling in the rat cardiomyocyte. This model combines equations from the Pandit electrophysiological model and Hinch model of calcium handling with equations describing myofilament mechanical activity from the 'Ekaterinburg-Oxford' mathematical model. The model reproduces both fast and slow responses to mechanical interventions in rat myocardium.
\end{abstract}

\section{Introduction}

As we know, the only Pandit-Hinch-Niederer (PHN) mathematical model has been developed to simulate excitation-contraction coupling (ECC) in the rat cardiomyocytes so far [1]. Particularly, the PHN model is able to reproduce the slow force response (SFR) to myocardium stretch. To simulate SFR, the authors needed to introduce an additional description of stretch-activated channels in the Pandit model of the rat Action Potential (AP), that were not accounted for in the original Panditmodel [2].

Significant limitation of the PHN is incapacity to simulate quick effects of the length changes, including Mechano-Calcium and Mechano-Electric Feedback effects (MCMEF) manifesting themselves during the twitches of cardiac muscles in a number of well-known experimental phenomena such as the load-dependence of relaxation and quick deformations [3]. We tried and overcame this limitation in our new model presented in this work.

It is very important to develop a model which takes into account mechanisms underlying such quick responses to the mechanical interventions, because these can contribute essentially to the appearance of functional disturbances in the cells, arrhythmia included [4-6].
Most clearly, the MCMEF effects were registered in experiments on rapid stretching/shortening during the contractile twitch in the cat and ferret heart muscles $[3,7$, 8]. Experiments in the rat preparations also showed similar results [9].

Cooperative influence of the attached crossbridge $(\mathrm{Xb})$ concentration on calcium - troponin $\mathrm{C}\left(\mathrm{Ca}^{2+}-\mathrm{TnC}\right)$ kinetics is one of the basic mechanisms contributing to MCMEF. Particular mechanisms underlying the length effects on the $\mathrm{Xb}$ attachment are being extensively debated. For a long time, the lattice spacing concept was prevailing one, suggesting that a decrease in the sarcomere inter-filament distance due to the cardiomyocyte lengthening facilitates actomyosin interactions and thus increases the probability of the $\mathrm{Xb}$ attachment [10, 11]. Recent experimental studies unraveled an essential role of the giant protein titin in the length dependence of the $\mathrm{Xb}$ dynamics $[12,13]$. In our model, the length-dependent probability for $\mathrm{Xb}$ binding is described phenomenologically as an overall result of all possible underlying molecular mechanisms. Together with the above cooperativity this length-dependent probability allows the EO-model to reproduce most of the MCMEF effects registered in the heart muscle.

\section{Methods}

Our new model of ECC in the rat cardiomyocyte combines the Pandit electrophysiological model [2] and Hinch model of calcium handling [14] with the model of the mechanical activity we early developed for the 'Ekaterinburg-Oxford' electro-mechanical mathematical model of mammalian cardiomyocytes (EO-model) [15]. EO-model describes ECC in the cardiomyocytes of several species of laboratory animals (e.g. rabbit, guinea pig), being parametrically adapted to each particular one. However, EO-model cannot be applied to simulate adequately the rat AP due to the specific features of the latter. Incorporation of the Pandit-Hinch model of the rat cardiomyocyte electrophysiology is a way to overcome this drawback. Mechanisms of cooperativity of regulatory and contractile proteins is a key feature of our module of 
the mechanical activity. The tuning of the parameters in the mechanical module of the integrative electromechanical rat model was performed on experimental data measured in the rat cardiac muscles [16]. We will refer to the combined rat model as
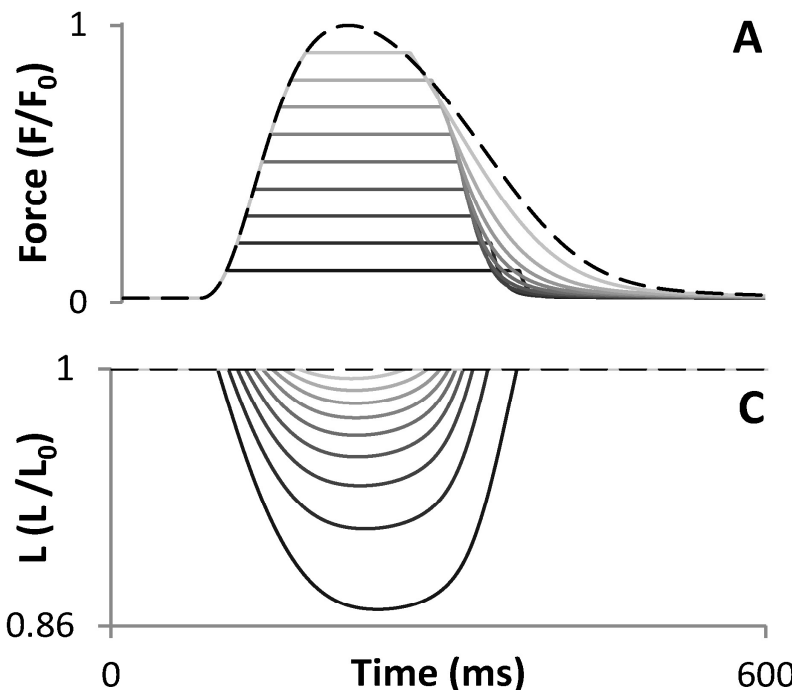

to $\mathrm{PH}+\mathrm{E}$ model.

The next section 'Results' demonstrates that our new $\mathrm{PH}+\mathrm{E}$ model of the rat cardiomyocytes ECC also is able to simulate these effects (including the load-dependence of relaxation).
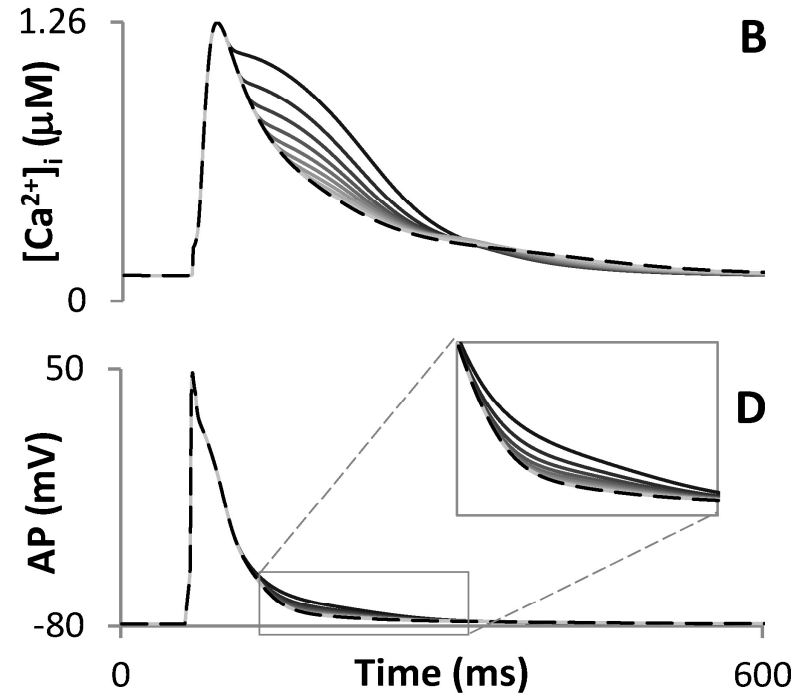

Figure 1. Load-dependence of the electro-mechanical activity in the virtual rat muscle. Simulation of series of myocardium contraction-relaxation cycles including an isometric twitch (dashed line) and isotonic twitches under different afterloads (shades of grey). A, C: Isometric/isotonic force of a virtual cardiomyocyte developed at an initial length $\left(0.9 \mathrm{~L}_{\max }\right)$ and isotonic shortening-lengthening at different afterloads. $\mathrm{B}, \mathrm{D}$ : respective $\mathrm{Ca}^{2+}$ transients, and action potentials.
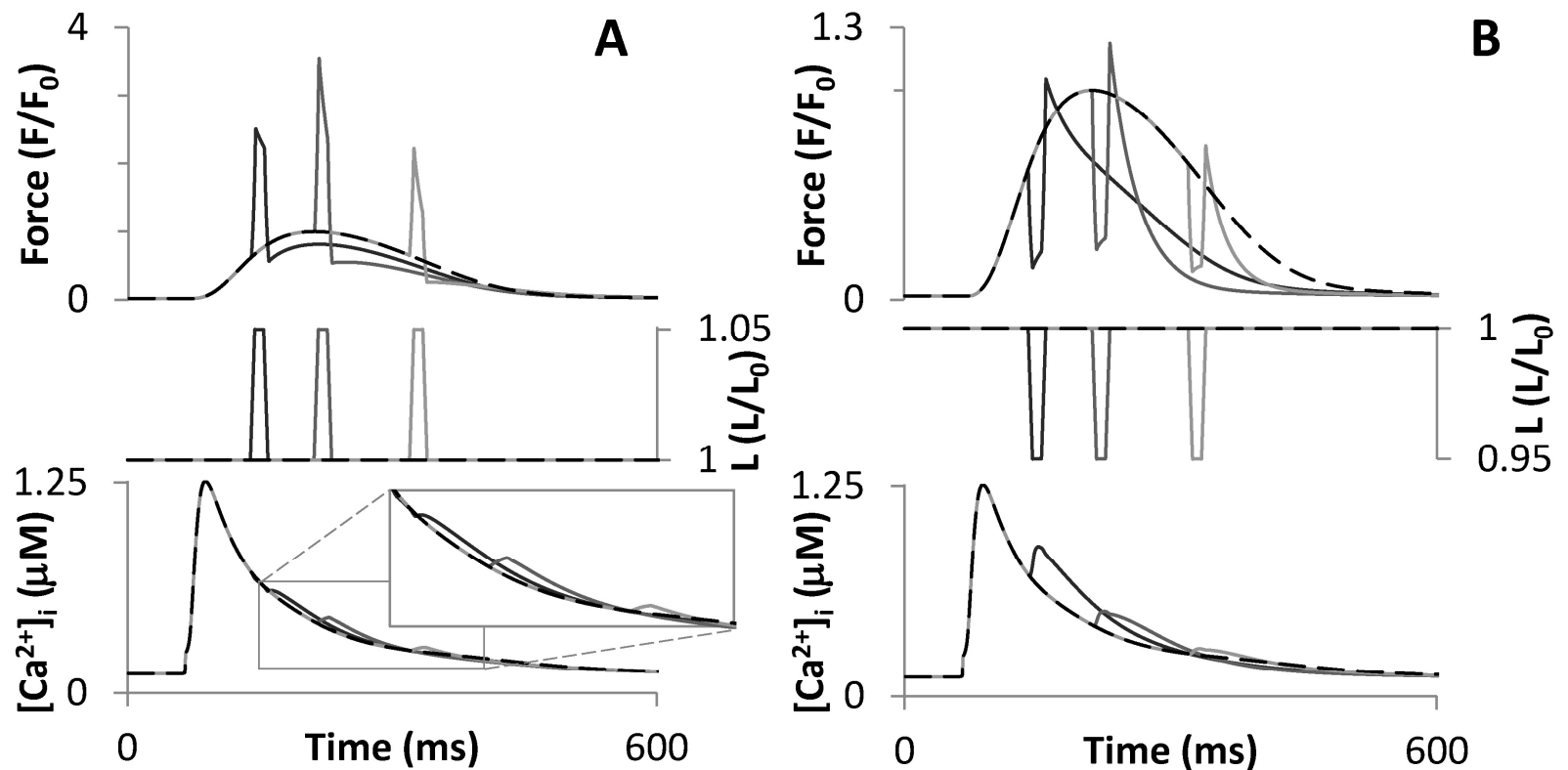

Figure 2. Quick responses to mechanical deformations. Force (top panels), length (medium panels), and $\left[\mathrm{Ca}^{2+}\right]_{\mathrm{i}}$ (bottom panels) changes in response to quick cyclic deformations of the virtual sample. Initial length is $0.9 \mathrm{~L}$ max. $5 \%$ stretchrelease deformations (A) and 5\% release-stretch deformations (B) are imposed for $20 \mathrm{~ms}$ at different phases of the isometric twitch $(80,150$, and $260 \mathrm{~ms}$ after the contraction onset). 
A

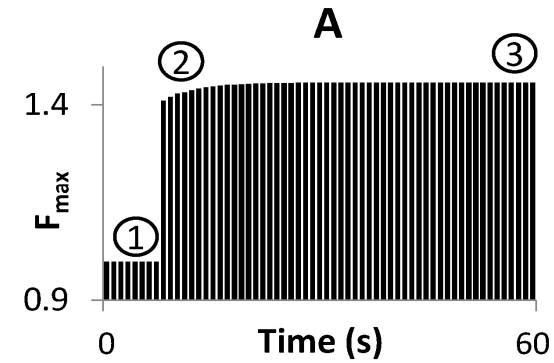

B

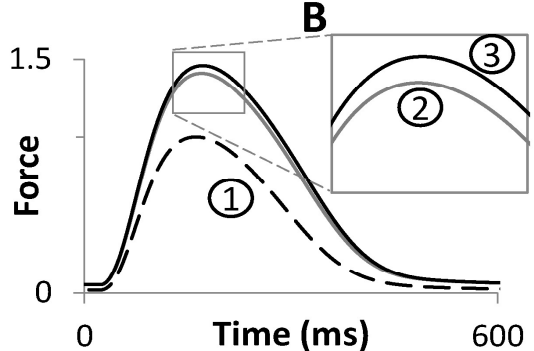

C

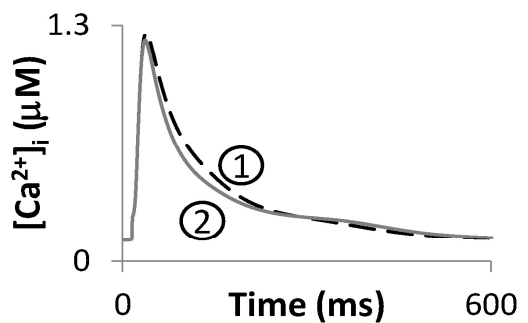

Figure 3. Slow force response to stretch in the virtual rat myocardium. A: Development of the isometric force at the initial length $\mathrm{L}_{0}=0.9 \mathrm{~L}_{\max }$ (phase 1), immediately after a $5 \%$ stretch (phase 2), and during the slow transition to the new steady state at the longer length (phase 3). Force is normalized to its peak before the stretch. B: The force development during isometric twitches typical for the phases (1) - (3) of the slow force response. C: Crossover of the $\mathrm{Ca}^{2+}$ transients of the phases (1) and (2).

\section{Results}

Results described below were obtained in $\mathrm{PH}+\mathrm{E}$, when simulating various mechanical conditions of contractions (isometric mode, afterloaded contractions and responses to quick deformations). Figure 1 shows simulations of afterloaded contractions under different afterloads.

We see that the mechanical behavior of the model is similar to experimental data [16]. The end-systolic shortening increases in the virtual muscle sample, while the velocities of shortening and lengthening increase with a decrease in afterload (Fig. 1C). A specific feature which is reproduced by the $\mathrm{PH}+\mathrm{E}$ model is the load-dependent shrinking of isotonic phase of the contraction-relaxation in comparison with isometric twitch duration (myofilament inactivation by shortening). The above features of the afterloaded contraction-relaxation cycles reflect the well-known phenomena of load-dependence of myocardial twitches. $\mathrm{PH}+\mathrm{E}$ model also produces the longer $\mathrm{Ca}^{2+}$ transients and APs (Fig. 1B, 1D) for the smaller afterloads in concordance with physiological experiments [3, 17]. The mechanisms underlying this load-dependence are the cooperative mechanodependence of myofilament $\mathrm{Ca} 2+$ activation which is accounted for in the mechanical part of the model and have been analyzed in our earlier works [18-21].

One more effect simulated in the $\mathrm{PH}+\mathrm{E}$ model and related to MCMEF is shown in Fig. 2. Results of quick mechanical deformations at different time moments of the simulated isometric twitch are similar to the data registered in vivo $[3,7,8]$. Two types of the deformation cycle are simulated here: a stretch-release (Fig. 2A) and release-stretch (Fig. 2B). The stretch of the virtual preparation during isometric contraction (Fig. 2A, L) under the first protocol didn't change $\left[\mathrm{Ca}^{2+}\right]_{\mathrm{i}}$ significantly, while the following quick release back to the initial length led to the significant inactivation: acceleration of the $\mathrm{Ca}^{2+}-\mathrm{TnC}$ dissociation that caused reduced subsequent isometric force development (Fig. 2A, F). The faster $\mathrm{Ca}^{2+}-\mathrm{TnC}$ decay also resulted in a surplus of the free cytosolic $\mathrm{Ca}^{2+}$ (Fig. 2A, $\left[\mathrm{Ca}^{2+}\right]_{i}$ ). The inverted sequence of the quick release-stretch (Fig. 2B, L) caused even more pronounced inactivation manifesting itself in both active force (Fig. 2B, F) and $\mathrm{Ca}^{2+}$ (Fig. $2 \mathrm{~B},\left[\mathrm{Ca}^{2+}\right]_{\mathrm{i}}$ ) signals. This more pronounced effect is a result of two inactivation factors superimposed: the decrease in the sarcomere length and an increase in the shortening velocity during the deformation, while the only latter factor contributes to the inactivation in the case of the stretch-release.

Note that the applied deformations weakly affected AP duration in the $\mathrm{PH}+\mathrm{E}$ model, unlike our previous simulations in the EO-model for larger animals, and unlike the data of the real experiments with these larger animals $[3,7,8]$. This distinction arises because the rat AP is much shorter and the mechanical deformations occur when the membrane is almost repolarized.

Along with simulation of these and other experiments with quick mechanical deformations during the myocardium twitches, $\mathrm{PH}+\mathrm{E}$ model reproduces the SFR effect in the series of isometric contractions following sustained myocardium stretch $[22,23]$. Noteworthy, that unlike the PHN model, $\mathrm{PH}+\mathrm{E}$ model reproduces this effect (Fig. 3) without taking into account stretch activated channels. Nevertheless, this seems reasonable to introduce such channels in the $\mathrm{PH}+\mathrm{E}$ as well to achieve quantitatively stronger SFR. The $\mathrm{PH}+\mathrm{E}$ shows not only secondary slow gradual increase in the peak isometric force after the stretch (Fig. 3, top) and accompanying drop in amplitude of $\left[\mathrm{Ca}^{2+}\right]_{\text {i }}$, but also the crossover of $\mathrm{Ca}^{2+}$ transients in the preparation before and right after the stretch. These results are similar to the experimental data from Kentish and Wrzosek [23].

\section{Conclusion}

The PH-E model of the excitation-contraction coupling 
is able to reproduce both fast MCMEF effects and slow force response in the rat myocardium. This model may be used to analyze and distinguish specific features of ECC in rat myocardium revealed in physiological experiments.

\section{Acknowledgements}

The work is performed in the frameworks of IIP UrB RAS projects (Nos. AAAA-A18-118020590031-8, AAAA-A18-118020590134-6), and supported by RFBR (No. 18-01-00059), by Act 211 Government of the Russian Federation, contract No. 02.A03.21.0006.

\section{References}

[1] Niederer SA, Smith NP. A mathematical model of the slow force response to stretch in rat ventricular myocytes. Biophysical Journal 2007;92(11):4030-44.

[2] Pandit SV, Clark RB, Giles WR, Demir SS. A mathematical model of action potential heterogeneity in adult rat left ventricular myocytes. Biophysical Journal 2001;81(6):3029-51

[3] Lab MJ, Allen DG, Orchard CH. The effects of shortening on myoplasmic calcium concentration and on the action potential in mammalian ventricular muscle. Circ Res 1984;55(6):825-9.

[4] Kohl P, Hunter P, Noble D. Stretch-induced changes in heart rate and rhythm: clinical observations, experiments and mathematical models. Progress in Biophysics and Molecular Biology 1999;71(1):91-138.

[5] Franz MR. Mechano-electrical feedback. Cardiovascular Research 2000;45(2):263-6.

[6] Calaghan SC, White E. The role of calcium in the response of cardiac muscle to stretch. Progress in Biophysics and Molecular Biology 1999;71(1):59-90.

[7] Lab MJ. Contraction-excitation feedback in myocardium. Physiological basis and clinical relevance. Circ Res 1982;50(6):757-66.

[8] Kurihara S, Komukai K. Tension-dependent changes of the intracellular $\mathrm{Ca} 2+$ transients in ferret ventricular muscles. The Journal of Physiology 1995;489 ( Pt 3):61725.

[9] Wakayama Y, Miura M, Sugai Y, Kagaya Y, Watanabe J, ter Keurs HE, et al. Stretch and quick release of rat cardiac trabeculae accelerates $\mathrm{Ca} 2+$ waves and triggered propagated contractions. American Journal of PhysiologyHeart and Circulatory Physiology 2001;281(5):H2133-42.

[10] Mcdonald KS, Moss RL. Osmotic compression of single cardiac myocytes eliminates the reduction in $\mathrm{Ca}^{2+}$ sensitivity of tension at short sarcomere-length. Circ Res 1995;77(1):199-205.

[11] Wang YP, Fuchs F. Osmotic compression of skinned cardiac and skeletal muscle bundles: effects on force generation, $\mathrm{Ca}^{2+}$ sensitivity and $\mathrm{Ca}^{2+}$ binding. $\mathrm{J}$ Mol Cell Cardiol 1995;27(6):1235-44.

[12] Mateja RD, Greaser ML, de Tombe PP. Impact of titin isoform on length dependent activation and cross-bridge cycling kinetics in rat skeletal muscle. Bba-Mol Cell Res 2013;1833(4):804-11.

[13] Ait-Mou Y, Hsu K, Farman GP, Kumar M, Greaser ML, Irving TC, et al. Titin strain contributes to the FrankStarling law of the heart by structural rearrangements of both thin- and thick-filament proteins. P Natl Acad Sci USA 2016;113(8):2306-11.

[14] Hinch R, Greenstein JL, Tanskanen AJ, Xu L, Winslow RL. A simplified local control model of calcium-induced calcium release in cardiac ventricular myocytes. Biophysical Journal 2004;87(6):3723-36.

[15] Sulman T, Katsnelson LB, Solovyova O, Markhasin VS. Mathematical modeling of mechanically modulated rhythm disturbances in homogeneous and heterogeneous myocardium with attenuated activity of $\mathrm{Na}^{+}-\mathrm{K}^{+}$pump. Bulletin of Mathematical Biology 2008;70(3):910-49.

[16] Katsnelson LB, Nikitina LV, Chemla D, Solovyova O, Coirault C, Lecarpentier Y, et al. Influence of viscosity on myocardium mechanical activity: a mathematical model. Journal of Theoretical Biology 2004;230(3):385-405.

[17] Kaufmann RL, Antoni H, Hennekes R, Jacob R, Kohlhardt M, Lab MJ. Mechanical response of the mammalian myocardium to modifications of the action potential. Cardiovascular Research 1971;Suppl 1:64-70.

[18] Izakov VY, Katsnelson LB, Blyakhman FA, Markhasin VS, Shklyar TF. Cooperative effects due to calciumbinding by troponin and their consequences for contraction and relaxation of cardiac muscle under various conditions of mechanical loading. Circ Res 1991;69(5):1171-84.

[19] Katsnelson LB, Markhasin VS. Mathematical modeling of relations between the kinetics of free intracellular calcium and mechanical function of myocardium. $\mathrm{J}$ Mol Cell Cardiol 1996;28(3):475-86.

[20] Katsnelson LB, Markhasin VS, Khazieva NS. Mathematical modeling of the effect of the sarcoplasmic reticulum calcium pump function on load dependent myocardial relaxation. Gen Physiol Biophys 2000;19(2):137-70.

[21] Solovyova O, Vikulova N, Katsnelson LB, Markhasin VS, Noble PJ, Garny A, et al. Mechanical interaction of heterogeneous cardiac muscle segments in silico: Effects on $\mathrm{Ca} 2+$ handling and action potential. Int $\mathrm{J}$ Bifurcat Chaos 2003;13(12):3757-82.

[22] Allen DG, Kurihara S. The effects of muscle length on intracellular calcium transients in mammalian cardiac muscle. The Journal of Physiology 1982;327:79-94.

[23] Kentish JC, Wrzosek A. Changes in force and cytosolic $\mathrm{Ca} 2+$ concentration after length changes in isolated rat ventricular trabeculae. The Journal of Physiology 1998;506(2):431-44.

Address for correspondence.

Name. Leonid B. Katsnelson

Full postal address. 620041, Pervomayskaya st., 106, Ekaterinburg, Russia

E-mail address. leonidkatsnelson51@gmail.com 